\title{
A Rare Site of Parasitic Granuloma
}

\section{Kshitij Kirane', Raiba Deshmukh², Avishkar Barase³, Qaidzohar Kanchwala ${ }^{4}$, Rutik Gandhi ${ }^{5}$}

\author{
${ }^{1,5}$ Senior Resident, ${ }^{2}$ Assistant Professor, ${ }^{3}$ Associate Professor, ${ }^{4}$ Senior Resident, \\ Department of General Surgery, Symbiosis Medical College for Women \& Symbiosis University Hospital and \\ Research Centre
}

Corresponding Author: Raiba Deshmukh

\begin{abstract}
Parasitic infection is common infestation encountered.

Poor hygiene, barefoot walking, sexual contact, consumption of uncooked food, contact with infected feces are the modes of transmission of parasite in a human Body

Parasitic infection may lead to Anemia, malnourishment, generalized weakness ${ }^{(1)}$
\end{abstract}

Key Words: Filarial Nematode, Anthelmintic drugs.

\section{INTRODUCTION}

Parasitic disease is most commonly transmitted by Parasitic Infestation. Parasites mostly invade the body through skin and mouth. Close contact with animals can also lead to parasitic infestation.

\section{CASE}

30 year old patient came to OPD with a complain of swelling below the left eye. Patient was apparently alright 1 month back when he started noticing a small swelling below his left eye. It was gradual in onset and size of swelling gradually increased.

There was no history of pain, fever, and discharge through swelling. stable.

On examination his vitals were

Local examination revealed approximately $2 \times 2 \mathrm{~cm}$ swelling on tear trough area below eye of left side of face. It was firm in consistency. No local rise of temperature. There was no discharge from swelling. It was non tender on palpation.

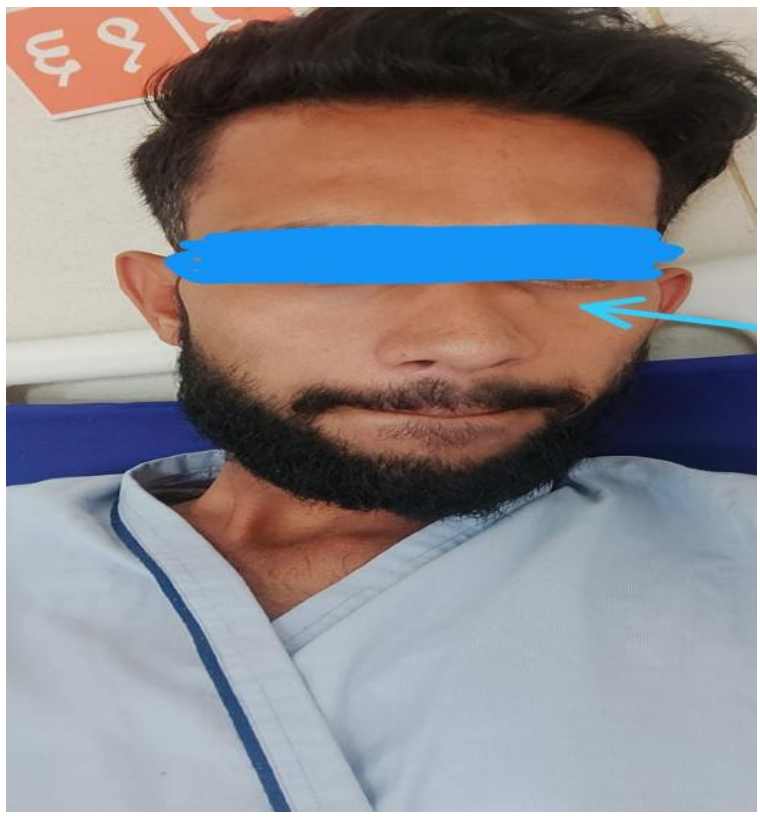

His routine OPD basis investigations such as CBC, HIV, HbsAg, HCV, BSL(R) was sent. All Investigations were within normal Limits.

Excision of swelling was planned under Local Anaesthesia.

Patient was given supine position in minor OT. Painting and drapping of affected area was done. $5 \mathrm{ml}$ of $2 \%$ lignocaine was injected around the affected area under aseptic precaution. Incision over swelling 
was taken. Granuloma was visualized and was surrounded by tuft of small tortuous capillaries. Granuloma was excised sparing the blood vessels. Hemostasis was maintained. Antiseptic solution followed by saline wash was given. All the layers of skin were sutured with 4-0 ethilon and dressing was done.

Specimen was sent for histopathology

Histopathology report revealed parasitic granuloma suggestive of filarial Nematode.

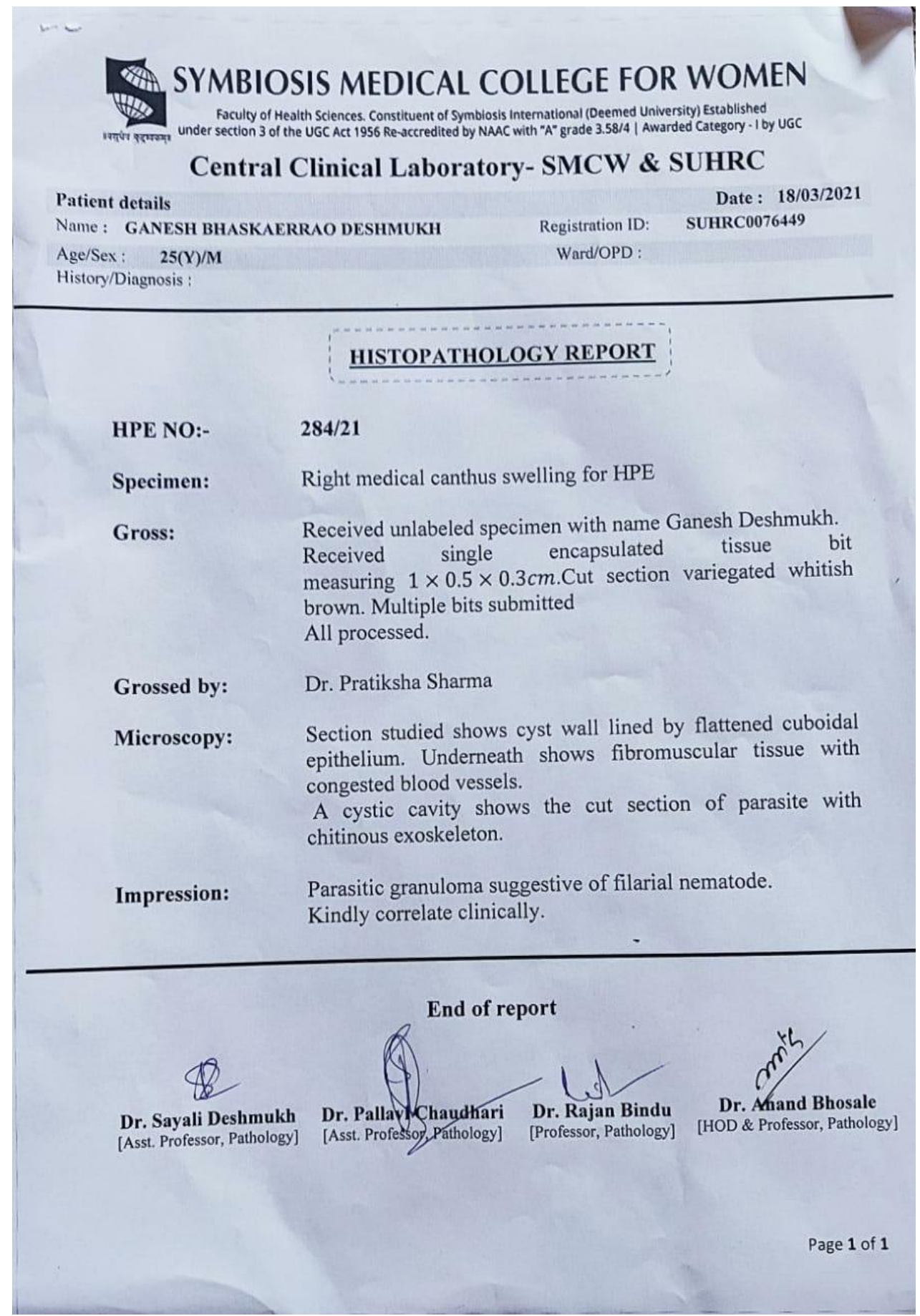

Patient was discharged on the next day of procedure along with oral Antibiotics, antacid and analgesic drug for 7 days. On $8^{\text {th }}$ post-operative day sutured were removed with no signs of infection. 


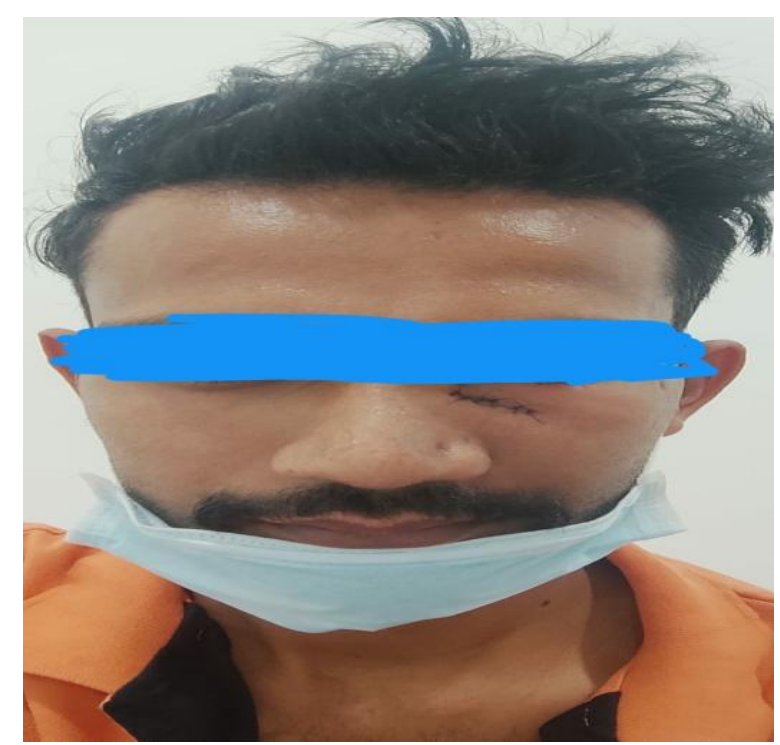

After the histopathology report confirmation patient was given a single dose of anthelmintic drug.

Patient has no complains and was vitally stable after suture removal

\section{DISCUSSION}

Parasitic infestation followed by granuloma formation occurs in response to chronic inflammation ${ }^{(2)}$. Early intervention is essential to avoid complications like abscess formation and deeper invasion of parasite. Proper postoperative management is important to avoid surgical site infection.

\section{CONCLUSION}

Parasitic Granuloma requires surgical intervention followed by adequate postoperative antibiotic and anthelmintic drugs with proper follow-up ${ }^{(3)}$. Diagnosis is done with clinical correlation and pathological analysis.

\section{Acknowledgement: None}

\section{Conflict of Interest: None}

\section{Source of Funding: None}

\section{REFERENCES}

1. Harrison's manual of medicine. Harrison, Tinsley Randolph, 1900-1978., Kasper, Dennis L.,, Longo, Dan L. (Dan Louis), 1949-, Fauci, Anthony S., 1940-, Hauser, Stephen L.,, Jameson, J. Larry (19th ed.). New York. 2016-05-22.

2. Williams, Olivia; Fatima, Saira (5 April 2020). "Granuloma". StatPearls. Treasure Island: StatPearls Publishing.

3. Kappagoda, Shanthi; Singh, Upinder; Blackburn, Brian G. (2011). "Antiparasitic Therapy". Mayo Clin. Proc. 86 (6): 561583.

How to cite this article: Kirane K, Deshmukh R, Barase A et.al. A rare site of parasitic granuloma. Int $J$ Health Sci Res. 2021; 11(7):290-292. DOI: https://doi.org/10.52403/ ijhsr.20210739 